\title{
Elastic step interactions on vicinal surfaces of fcc metals
}

\author{
R. Najafabadi a,*, D.J. Srolovitz ${ }^{\text {a,b }}$ \\ " Department of Materials Science and Engineering, University of Michigan, Ann Arbor, MI 48109, USA \\ ${ }^{\mathrm{b}}$ Department of Materials and Interface, Weizmann Institute of Science, Rehovot 76100, Israel
}

Received 16 February 1994; accepted for publication 23 May 1994

\begin{abstract}
The structural and energetic properties of [100] and [110] steps on the (001) surface of fcc metal have been determined by $T=0$ atomistic simulations. The interactions between [100] steps and between [110] steps on the (001) surface are determined from the surface energy of a series of $(01 n)$ and $(11 \mathrm{~m})$ surfaces, respectively. For step spacings larger than three fcc lattice parameters $\left(R>3 a_{0}\right)$, we find that the interaction energy between two similar steps on the (001) surface can be reasonably represented by the functional form $R^{-2}$, in agreement with the prediction of a simple linear elastic analysis based upon a line dipole force model of a step. However, we observe qualitative differences between the displacement fields determined by the two methods. For $R<3 a_{0}$, on the other hand, we find that the interaction between steps deviates significantly from the form $R^{-2}$. These deviations demonstrate that both dipole and quadrupole force distributions are necessary to account for step-step interactions for spacings as small as a fraction of a lattice parameter up to infinite step spacings. We show that a [100] step on the (001) surface in $\mathrm{Au}$ and $\mathrm{Pt}$ (but not in $\mathrm{Ag}, \mathrm{Au}, \mathrm{Cu}$, or $\mathrm{Pd}$ ) may lower the surface energy by transforming into a zig-zagged [110] step.
\end{abstract}

\section{Introduction}

The structural and energetic properties of steps on an otherwise flat surface are important in understanding surface related phenomena, such as epitaxial growth, surface roughening, and equilibrium crystallite shapes. Additionally, the interaction between steps has a strong influence on the spatial and temporal evolution of the surfaces. Step-step interactions arise mainly from the interaction between the elastic displacement

\footnotetext{
* Corresponding author. E-mail: reza@engin.umich.edu; Fax: +13137634788 .
}

fields within the solid which are associated with the individual steps. Since the step displacement field decays as $1 / R$ away from the steps, the step-step interaction energy decays slowly with step separation.

Theoretical calculations [1], based on continuum elasticity, predict that the step-step interaction energy decays as the square of the inverse step separation. In that study, the displacement field associated with a step on a flat surface was modeled as a line of dipole forces directed along the surface and acting perpendicular to the step direction. The strength of the dipole force is related to the surface tension and the elastic properties of the bulk material. Other studies have employed atomistic simulation methods [2,3] 
to determine the structure and energy of steps and to test [3] the validity of the continuum elasticity prediction [1]. Since the displacement fields decay very slowly away from the step, large systems must be employed in atomistic simulations. Alternatively, step-step interactions can be examined via atomistic simulations by employing arrays of steps, such that the elastic fields of the individual steps screen each other.

In a previous study of [110] steps on a (001) copper surface [2] using a Morse pair potential, the atomic relaxation was limited to the first two (002) atomic planes at the surface. Furthermore, fixed boundary conditions were employed parallel to the step direction in the (001) plane. Since these constraints were imposed on the simulation cell due to limited computational resources available twenty years ago, those numerical results cannot be used to predict the detailed nature of step-step interactions. Recently, another simulation study [3] examined the properties of [100] steps on both the (001) and (011) surfaces in gold and copper. That study concluded that the stepstep interaction varies with the square of the inverse step separation, in agreement with the continuum elasticity analysis [1]. A similar conclusion [4] was reached for the [100] steps on the (001) surface of silicon. Although the elastic analysis was based upon continuum ideas, it was found that the inverse square dependence of the step-step interaction energy with step separation was valid for step separations as small as one lattice constant [3]. This result is surprising given the over simplification of the true atomic displacement field assumed in the continuum theory, especially on length scale comparable to the discreteness of the atomic lattice. The goal of the present study is to examine step - step interactions over a wide range of step spacing, from very large, where the continuum theory is expected to be valid, to very short, where the discreteness of the atomic lattice is important and the assumptions of the continuum theory break down.

In the present study, we investigate the structural and energetic properties of [100] and [110] steps on the (001) surface of six fcc metals: Ag, $\mathrm{Au}, \mathrm{Cu}, \mathrm{Ni}, \mathrm{Pd}$, and $\mathrm{Pt}$. To carry out this investigation, we employed the static energy minimiza- tion method $(T=0)$ and EAM potentials [5] to represent the interatomic interactions. The interactions between [100] steps and between [110] steps on the (001) surfaces were determined from a series of simulations performed on $(01 n)$ surfaces $(n<71)$ and on $(\overline{1} 1 m)$ surfaces $(m<41)$, respectively. In the next section, we describe our computational procedure and the geometry employed in the simulations. In Section 3, we derive an expression relating the step-step interaction energy, step energy, and surface energy, within the framework of linear elasticity. This expression is used to extract these quantities from the simulation data. In Section 4, we present the surface energies for the vicinal $(01 \mathrm{n})$ and $(11 \mathrm{~m})$ surfaces and the interaction energies between [100] steps and between [110] steps on the (001) surface of six fcc metals. Finally, in Section 5, we compare our results for the step-step interaction energies and strain fields with those from the continuum elasticity analysis, and discuss the relative stability of the [100] steps on the (001) surfaces of these metals.

\section{Simulation method}

In order to determine the interaction energies between [100] steps and between [110] steps on the (001) surface, we calculated the surface energies of a series of $(01 n)$ and $(\overline{1} 1 m)$ surfaces. We employed the static energy minimization method $(T=0)$ and EAM potentials [5] to represent the interatomic interactions in the fcc metals: $\mathrm{Ag}$, $\mathrm{Au}, \mathrm{Cu}, \mathrm{Ni}, \mathrm{Pd}$, and Pt. These surfaces are shown schematically in Fig. 1 for $n=4$ and $m=5$. As in our previous simulations [6], we applied periodic boundary conditions in both the $X([0 n \overline{1}])$ and $Y$ ([100]) directions for the $(01 n)$ surface, and the $X$ $([m \bar{m} 2])$ and $Y([110])$ directions for the $(\overline{1} 1 \mathrm{~m})$ surface. In the $-Z$ direction, we employed fixed boundary conditions, by attaching the computational cell to a perfect fcc crystal with the appropriate zero temperature lattice parameter. During the course of the energy minimization, the size of the computational cell in the $-Z$ direction was extended such that the surface energy remained unchanged upon further extension (to 
within $<10^{-6} \mathrm{~mJ} / \mathrm{m}^{2}$ ) and the forces on all atoms were less than $10^{-5} \mathrm{eV} / \AA$. We note that the final size of the computational cell in the $-Z$ direction increases with increasing $n$ and $m$ for the $(01 n)$ and $(\overline{1} 1 m)$ surfaces, respectively, since the decay length of the displacement field of the periodic array of steps scales inversely with step spacing and the step spacing increases with increasing $n$ or $m$ [7]. The total number of atoms required to satisfy the above energy convergence criterion were approximately 30000 and 24000 for the longest period $(01 \mathrm{n})$ and $(\overline{1} 1 \mathrm{~m})$ surfaces studied, respectively $(n=71, m=41)$.

\section{Elasticity analysis}

The $(01 n)$ and $(\overline{1} 1 m)$ surfaces (with odd $m$ ) may be decomposed into (001) terraces and arrays of the equally spaced [100] steps and [110]

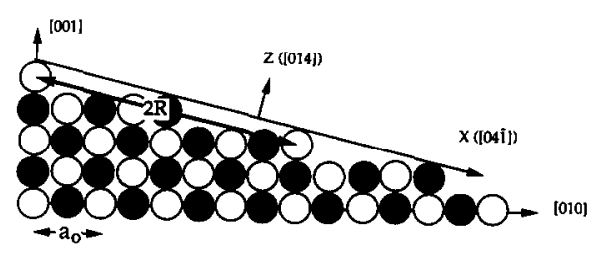

(a)

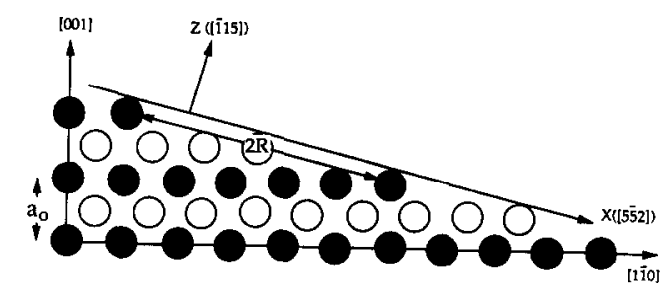

(b)

Fig. 1. A schematic representation of the (014) and (115) surfaces. The open and filled circles represent the two different stackings of (a) (010) planes and (b) (110) planes. Periodic border conditions were employed in the $X$ and $Y$ directions. The $Y$ direction corresponds to the [010] direction for the $(01 n)$ surfaces and to the [110] for the (11 $m$ ) surfaces. In the $Z$ direction a fixed border condition was used. $a_{0}$ and $R$ are the lattice constant and step spacing, respectively. steps, respectively (as shown in Fig. 1). The (01n) surface energy, $\gamma_{01 n}$ may be expressed as

$R \gamma_{01 n}=\gamma_{\text {step }}^{[100]}+n \frac{a_{0}}{2} \gamma_{001}+\gamma_{\text {int }}(R)$,

where $\gamma_{\text {step }}^{[100]}$ and $\gamma_{001}$ represent the [001] step energy per unit length and the (001) surface energy per unit area, respectively, and $n a_{0} / 2$ is the terrace length ( $a_{0}$ is the cubic unit cell lattice constant). The [100] step spacing, $R$, is $\left(a_{0} / 2\right)$ $\sqrt{n^{2}+1}$. The third term in Eq. (1), $\gamma_{\text {int }}(R)$, represents the contribution to the $(01 n)$ surface energy from the interactions between the [100] steps. This equation relates $\gamma_{\mathrm{step}}^{[100]}$ and $\gamma_{\text {int }}(R)$ to the $(001)$ and $(01 n)$ vicinal surface energies, which may be determined from atomistic simulation studies. Similarly, the $(11 \mathrm{~m})$ surface energy may be written as

$R \gamma_{11 m}=\gamma_{\text {step }}^{[110]}+m \frac{a_{0}}{2 \sqrt{2}} \gamma_{001}+\gamma_{\mathrm{int}}(R)$

where $R=\left(a_{0} / 2\right) \sqrt{1+m^{2} / 2}$.

Since the strains due to the steps are small throughout the solid, the step-step interactions may be analyzed within the framework of linear elasticity. The strain field $\epsilon$ associated with the steps is simply the sum of the strains associated with the individual steps, $\epsilon_{\text {total }}=\Sigma \epsilon_{\mathrm{i}}$, where bold symbols indicate tensors or vectors. The total elastic energy of the system $W_{\text {total }}$ may be written as

$$
\begin{aligned}
W_{\text {total }}= & \frac{1}{2} \int \mathbf{C}\left[\sum_{i} \epsilon_{i}(r)\right]\left[\sum_{j} \epsilon_{j}(\boldsymbol{r})\right] \mathrm{d} V \\
= & \frac{1}{2} \int \mathbf{C}\left[\sum_{i} \epsilon_{i}(\boldsymbol{r}) \epsilon_{i}(\boldsymbol{r})\right] \mathrm{d} V \\
& +\frac{1}{2} \int \mathbf{C}\left[\sum_{i} \sum_{j \neq i} \epsilon_{i}(\boldsymbol{r}) \epsilon_{j}(\boldsymbol{r})\right] \mathrm{d} V \\
= & N\left[\gamma_{\text {step }}(R)+\sum_{j=1}^{\infty} \gamma_{\mathrm{s}-\mathrm{s}}(j R)\right] \\
= & N\left[\gamma_{\text {step }}(R)+\gamma_{\mathrm{int}}(R)\right],
\end{aligned}
$$

where $\mathbf{C}$ is the elastic constant tensor of the material, $\gamma_{\mathbf{s}-\mathbf{s}}$ is the interaction energy between a 
pair of steps and $\gamma_{\mathrm{int}}$ is the total interaction energy per step (i.e., one half of the interaction energy of one step with all other steps).

We expand the interaction energy, $\gamma_{\mathrm{s}-\mathrm{s}}(R)$ in an inverse power series in the step-step separation $R$, normalized by the lattice parameter

$\gamma_{s-\mathrm{s}}(R)=\sum_{k=1}^{\infty} \zeta_{k}\left(\frac{a_{0}}{R}\right)^{k}$,

where the coefficients $\zeta_{k}$ are material dependent and are to be determined. The total step interaction energy may now be written as

$$
\begin{aligned}
\gamma_{\mathrm{int}}(R) & =\sum_{j=1}^{\infty} \gamma_{\mathrm{s}-\mathrm{s}}(j R) \\
& =\sum_{j=1}^{\infty}\left[\sum_{k=1}^{\infty} \zeta_{k}\left(\frac{a_{0}}{j R}\right)^{k}\right] \\
& =\sum_{k=1}^{\infty}\left\{\zeta_{k}\left(\frac{a_{0}}{R}\right)^{k}\left[\sum_{j=1}^{\infty}\left(\frac{1}{j}\right)^{k}\right]\right\}
\end{aligned}
$$

or

$$
\gamma_{\text {int }}(R)=\sum_{k=1}^{\infty} \zeta_{k} \beta_{k}\left(\frac{a_{0}}{R}\right)^{k},
$$

where the material independent coefficients $\beta_{k}$ $=\sum_{j=1}^{\infty} j^{-\mathrm{k}}$ may be easily calculated: $\beta_{2}=\pi^{2} / 6$ $=1.645, \beta_{3}=1.202$, and $\beta_{4}=\pi^{4} / 90=1.082$. The coefficient $\beta_{1}$ is infinite since the sum over $j^{-1}$ diverges. However, since the total interaction en-

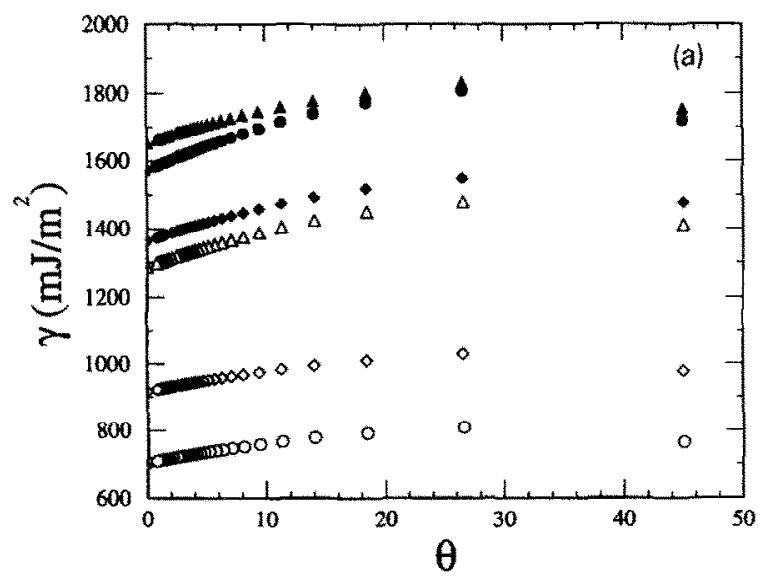

ergy between steps is finite, the coefficient $\zeta_{1}$ in Eq. (4) must vanish. Thus, substituting this for $\gamma_{i m t}$ in $\mathrm{Eq}$. (1), we obtain the following

$\gamma_{\text {step }}^{[100]}+\sum_{k=2}^{k_{\max }} \zeta_{k} \beta_{k}\left(\frac{a_{0}}{R}\right)^{k}=R \gamma_{01 n}-n \frac{a_{0}}{2} \gamma_{001}$,

where $k_{\max }$ is in principle infinity, but is finite in all practical implementations of the expansion. The step energy and the $\zeta_{k}$ 's may be determined by fitting Eq. (7) to the surface energy data (determined from the simulations) via a least-squares fitting procedure. A similar expression is found for the $(\overline{1} 1 \mathrm{~m})$ surfaces.

\section{Results}

\subsection{Surface energies}

The variation of the $(01 n)$ relaxed surface energies with the vicinal angle $\theta$ is shown in Fig. 2a for the six fcc metals $(\mathrm{Ni}, \mathrm{Cu}, \mathrm{Ag}, \mathrm{Pd}, \mathrm{Pt}$, and $\mathrm{Au}$ ), where the angle $\theta$ is defined as the angle between the $(01 n)$ and the $(001)$ planes $(\tan \theta=$ $1 / n$ ). All six curves show the same general trend: a minimum at $\theta=0(001)$ and a maximum at $\theta=25.56(012)$.

The difference between the surface energies of the equilibrium (relaxed) and bulk-terminated (unrelaxed) surface structures is the surface relaxation energy, $\Delta \gamma$. Fig. $2 b$ shows the variation

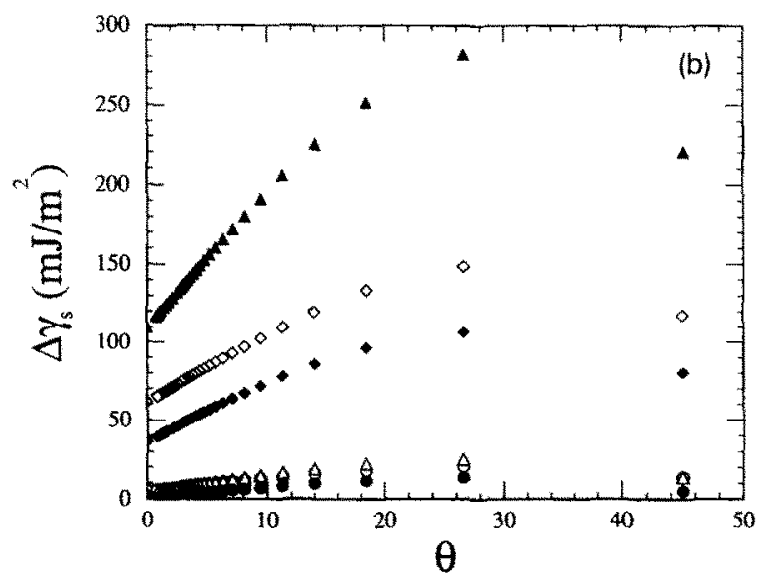

Fig. 2. The (a) (01n) relaxed surface energy and (b) $(01 n)$ surface relaxation energy as a function of the vicinal angle for six different fcc metals. The symbols $(\mathrm{O}),(\diamond),(\Delta),(\bullet),(\bullet)$, and $(\Delta)$ represent $\mathrm{Ag}, \mathrm{Au}, \mathrm{Cu}, \mathrm{Ni}, \mathrm{Pd}$, and $\mathrm{Pt}$, respectively. 
in the surface relaxation energy with the vicinal angle. The surface relaxation energy is the energy associated with the atomic displacements near the surface, measured relative to the bulkterminated structure. Fig. $2 \mathrm{~b}$ shows that the relaxation energies for the $(01 n)$ surfaces in $\mathrm{Ni}, \mathrm{Cu}$, and $\mathrm{Ag}$ are very small $\left(<20 \mathrm{~mJ} / \mathrm{m}^{2}\right)$ compared to those in Pd, Au and Pt. The variation of the surface relaxation energies with $\theta$ for $\mathrm{Pd}$ are $35-100 \mathrm{~mJ} / \mathrm{m}^{2}$, for $\mathrm{Au}$ are $60-150 \mathrm{~mJ} / \mathrm{m}^{2}$ and for Pt are $110-280 \mathrm{~mJ} / \mathrm{m}^{2}$. The ordering of the $(01 n)$ surface relaxation energies is consistent with those found for the flat (001) surfaces in $\mathrm{Ni}, \mathrm{Cu}$, $\mathrm{Ag}, \mathrm{Pd}, \mathrm{Au}$, and $\mathrm{Pt}$, which are 0.2, 3.0, 5.1, 36.6, 61.3 , and $109.7 \mathrm{~mJ} / \mathrm{m}^{2}$, corresponding to a change of $-0.006,-0.029,-0.039,-0.086,-0.117$, and $-0.123 \AA$, in the spacing between the top two (002) planes, respectively. Not surprisingly, the ordering of the relaxation energies is consistent with the ordering of the magnitude of the strain $\left(\epsilon_{z z}\right)$ at the surface.

Fig. 3 shows similar results obtained for the $(\overline{1} 1 \mathrm{~m})$ surfaces. The trends in the $(\overline{1} 1 \mathrm{~m})$ surface energies of the metals are similar to those found for the $(01 n)$ surfaces. The relaxation energies for the $(\overline{1} 1 \mathrm{~m})$ surfaces in $\mathrm{Ni}, \mathrm{Cu}$, and $\mathrm{Ag}$ are very small $\left(<20 \mathrm{~mJ} / \mathrm{m}^{2}\right)$ compared to those in $\mathrm{Pd}$, $\mathrm{Au}$, and $\mathrm{Pt}$ which range from approximately 35 to 90 , from 60 to 120 , and from 110 to $220 \mathrm{~mJ} / \mathrm{m}^{2}$, respectively.

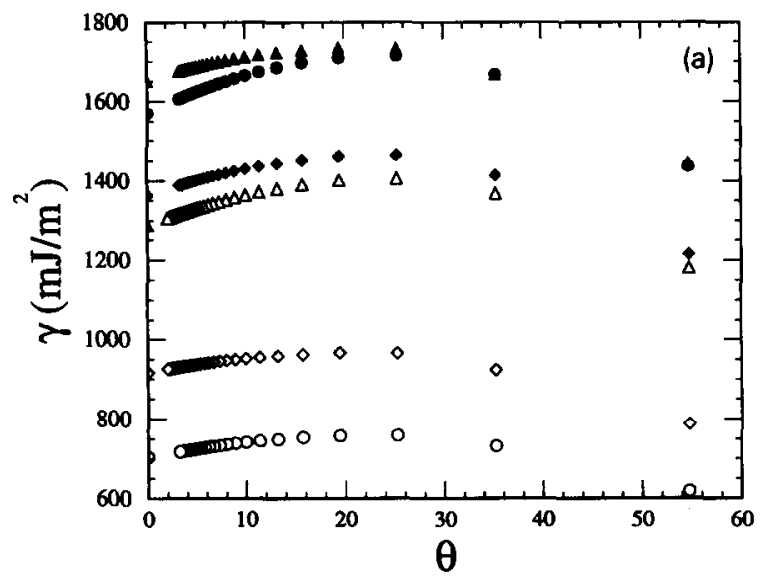

\subsection{Atomic displacements}

The atomic displacements associated with the presence of a [100] step on an otherwise flat (001) surface are defined as

$u_{\alpha}\left(r_{i}^{0}\right)=r_{i \alpha}^{01 n}-r_{i \alpha}^{001}$,

where $r_{i}^{0}$ is the position of atom $i$ on the unrelaxed $(01 n)$ surface, $r_{i \alpha}^{01 n}$ represents the $\alpha$ ([010] or [001]) component of the position of atom $i$ at a terrace on the relaxed $(01 n)$ surface and $r_{i \alpha}^{001}$ corresponds to the $\alpha$ component of the position of atom $i$ at the relaxed (001) flat surface. $r_{i \alpha}^{001}$ is determined by simply identifying the (002) plane in the flat (001) surface that corresponds to the (002) plane of the (001) terrace to which atom $i$ belongs. All atoms on an (002) plane of the flat (001) surface have the same displacements. Although the atoms close to the [100] steps may experience much larger displacements than the other atoms on the (001) terraces, there is no ambiguity in identifying their corresponding (002) planes on the flat (001) surface since the atomic displacements are very small compared with the (002) planar spacing. We also note that, due to the translational symmetry in the [100] direction (parallel to the step direction in the $(01 n)$ surface), the atomic displacements in the [100] direction are zero for both the $(01 n)$ and flat (001)

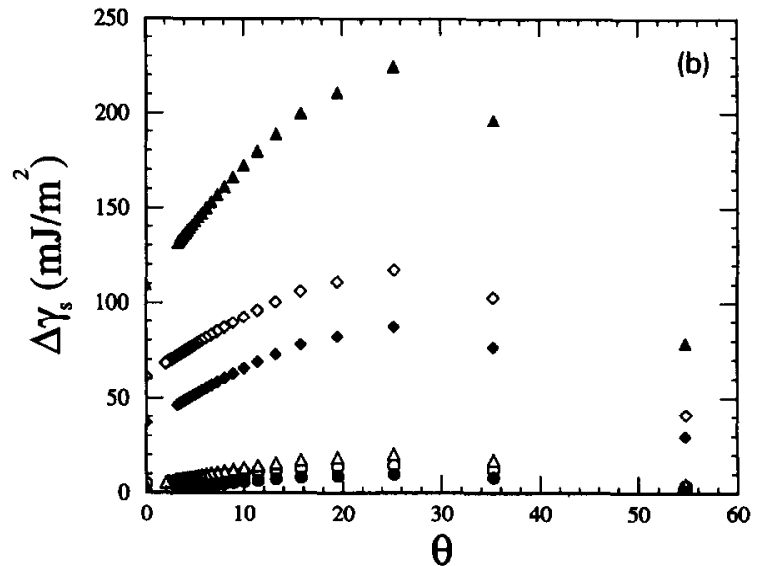

Fig. 3. The (a) $(\overline{1} 1 \mathrm{~m})$ surface energy and (b) $(\overline{1} 1 \mathrm{~m})$ surface relaxation energy as a function of the vicinal angle for six different fcc metals. The symbols $(\mathrm{O}),(\diamond),(\Delta),(\bullet),(\bullet)$, and $(\Delta)$ represent $\mathrm{Ag}, \mathrm{Au}, \mathrm{Cu}, \mathrm{Ni}, \mathrm{Pd}$, and $\mathrm{Pt}$, respectively. 
surfaces. An equivalent definition is used for the atomic displacements associated with the [110] steps on $(\overline{1} 1 \mathrm{~m})$ surfaces.

Fig. 4 shows the atomic displacements for atoms on or near the $(01 n)$ gold surface (projected onto the (100) plane) corresponding to $n=71,51$, and 31 . The displacements immedi-

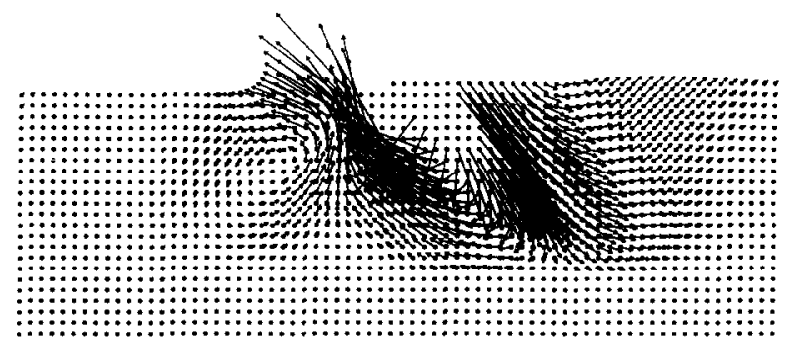

(a)

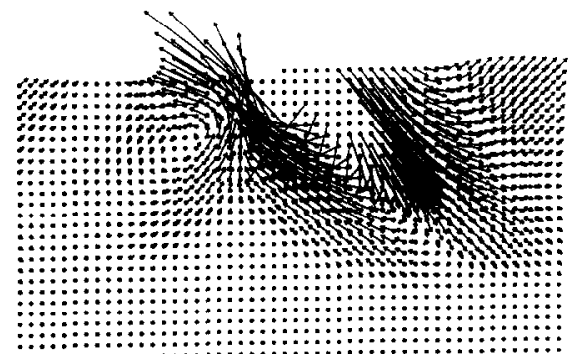

(b)

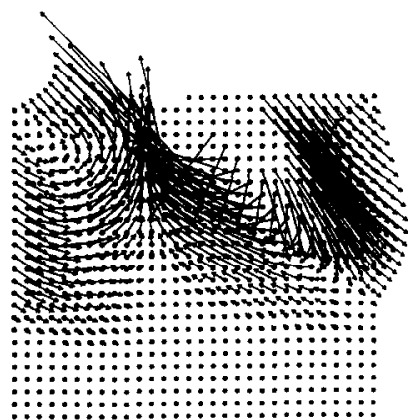

(c)

Fig. 4. Displacement vectors of atoms on and below one repeat unit of the $\mathrm{Au}(01 n)$ surfaces as projected on the $(010)$ plane corresponding to (a) $n=71$. (b) $n=51$, and (c) $n=31$. A [100] step of height $a_{0} / 2$ is seen in the center of each figure. The displacements in [010] are zero due to the translational symmetry in this direction. All displacements are magnified by a factor of $5 \times 10^{8}$. The large displacements of the atoms very near the step are not shown. ately adjacent to the steps are not shown in these figures, since these displacements are too large to be plotted for the magnification factor employed here and since our focus is the elastic displacement fields within the bulk. One clearly sees rotational components in the displacement fields close to the steps. These rotational components are obviously related to the presence of a torque associated with the step on the $(01 n)$ surfaces. Since no net torque exists on the sample (otherwise, the sample would rotate), this torque is balanced by two opposite rotational fields. As the spacing between the [100] steps on the (001) surface is varied from $35.5 a_{0}(n=71)$ to $15.5 a_{0}$ $(n=31)$, the displacement field around the steps remains essentially unchanged, as seen in Fig. 4. The atomistic displacement results for the surfaces of the other five fcc metals show qualitatively similar behavior as those observed for the Au(01n) surfaces. Since the displacement field around a step does not vary appreciably with step spacing, the observed displacement fields in the vicinity of a step are largely not associated with step-step interactions.

The [001] $(z)$ component of the atomic displacement on the upper terrace near the step is negative (inward relaxation) but gradually becomes positive (outward relaxation) with increasing distance from the step. There is a maximum in the outward relaxation between two steps (this is at approximately $11 a_{0}$ for the $(0171)$ Au surface shown in Fig. 4a). The maximum seems to disappear as the step spacing is decreased due to the interaction between the displacement fields (see Fig. 4c). In order to show the atomic displacements more quantitatively we have plotted (Fig. 5) both the [010] and [001] components of the atomic displacement for the atoms in the top (002) plane of the (001) terrace. The displacements in both directions do not vary monotonically with distance from the step and go through extrema as the distance away from the step is increased (or decreased from the step on the other side). Similar results were obtained for the $(01 n)$ surfaces of the other five fcc metals.

Fig. 6 shows how the magnitude of the atomic displacements $|\boldsymbol{u}|=\sqrt{u_{010}^{2}+u_{001}^{2}}$ midway be- 
tween two steps vary with distance into the solid, $d$. As for the displacements along the surface, the atomic displacement in the bulk do not decay monotonically with increasing distance $d$. The atomic displacements decay much faster for the (0130) than for the $(0170)$ gold surface with increasing distance $d$. This shows, in agreement with a linear elasticity calculation [7], that the displacement associated with large $n$ decays more slowly into the bulk than that associated with small $n$. In addition we find that for the same (0130) surface, the atomic displacements are considerably larger in $\mathrm{Au}$ than in $\mathrm{Cu}$. This is consistent with the magnitude of the atomic displacements associated with surface relaxation found for the flat (001) surfaces of $\mathrm{Au}$ and $\mathrm{Cu}$.

\subsection{Interaction between steps}

In order to determine the [100] step energy and the interaction energy between [100] steps on the (001) surface separated by a distance $R$, we fit Eq. (7) to the surface energy data determined from our simulations using a least-squares fitting procedure. In the fitting procedure, we only used the surface energies corresponding to the $(01 n)$

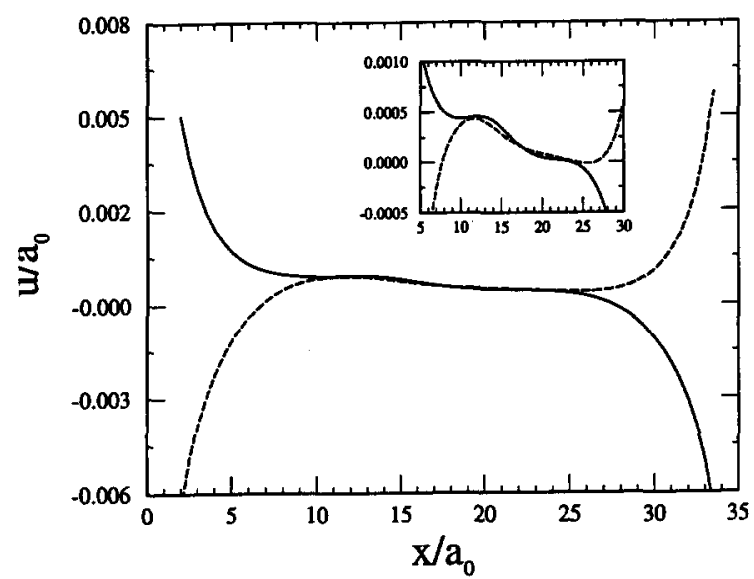

Fig. 5. Atomic displacement on the Au(0171) surface along the $x$ direction (see Fig. 1) as a function of distance away from the [100] step. The solid and dashed lines show the in-plane $(x)$ and normal $(z)$ displacements, respectively. The inset figure shows a magnified view of the displacements.

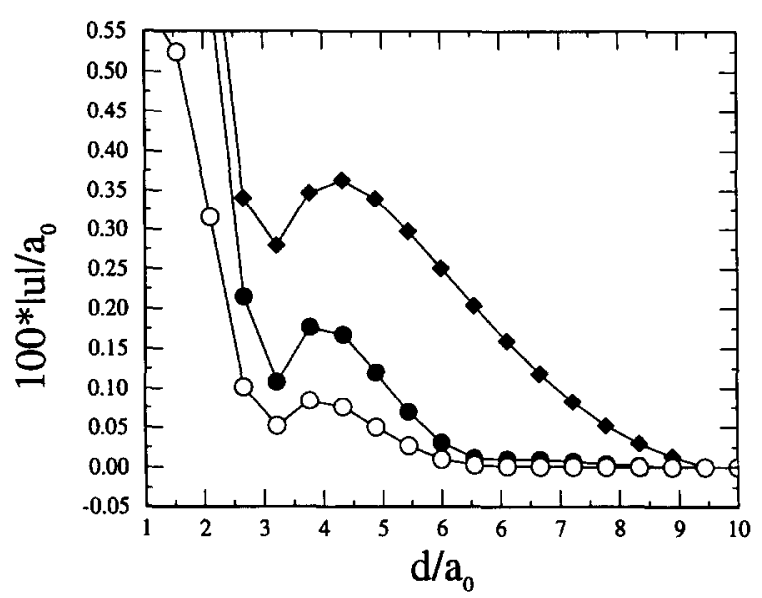

Fig. 6. The normalized and scaled displacements $100 \times|u| / a_{0}$ $\left(|u|=\left(u_{x}^{2}+u_{z}^{2}\right)^{1 / 2}\right)$ of atoms below the surface and on the (010) plane passing through the midpoint in a terrace of the $(01 n)$ surface as a function of distance from the surface, $d$. The symbols $(\bullet),(\bullet)$, and $(O)$ correspond to the displacements of the $\mathrm{Au}(0131), \mathrm{Au}(0171)$, and $\mathrm{Cu}(0131)$ surfaces, respectively. Note that the closer the steps are on the surface (smaller $n$ ) the faster the displacement decays in the bulk.

surfaces with $6 \leq n \leq 20$. We excluded the $n \leq 6$ $(01 n)$ surface energy data because the [100] steps on these surfaces are less than $3 a_{0}$ distance apart and, therefore, their interaction energy may not be accurately described using linear elasticity. When $n$ is large it is important to include displacements in the bulk far from the surface. Since we use fixed boundary conditions on the $-Z$ edge of the model, these displacements are not adequately described for large $n$. Therefore, the fitting procedure used in determining the stepstep interactions omitted the $(01 n)$ surfaces with $n>20$.

We performed several fits corresponding to truncating the power series in $R^{-k}$ at different values of $k_{\max }$ in Eq. (7). Table 1 shows the values of the $\zeta$ 's found by fitting and the associated goodness of fit parameter $\chi^{2}\left(\chi^{2}=0\right.$ indicates a perfect fit) for $k_{\max }=2,3$, and 4 . We find that $\chi^{2}$ is typically an order of magnitude smaller when Eq. (7) with $k_{\max }=3$ is employed rather than $k_{\max }=2$ for $\mathrm{Au}, \mathrm{Pd}$, and Pt. For $\mathrm{Ag}, \mathrm{Cu}$, and $\mathrm{Ni}$ the reduction in $\chi^{2}$ is at least $50 \%$ on 
going from $k_{\max }=2$ to $k_{\max }=3$. Table 1 also shows that $\chi_{2}$ is not significantly reduced by including more terms in the power series (i.e., $k_{\max }>3$ ). Table 1 shows that $\zeta_{2}$ does not vary significantly with $k_{\max }$. On the other hand, the magnitude of $\zeta_{3}$ changes by nearly a factor of 2 on increasing $k_{\max }$ from 3 to 4 . Therefore, the reported values of the coefficients of the $1 / R^{2}$ interaction energy are accurately determined and insensitive to the choice of $k_{\max }$, while the coefficients of the higher order terms are not reliably determined (i.e., they can only be accurately determined in the large $k_{\max }$ limit). Since $\zeta_{2}>0$ for all six elements, we conclude that widely separated [100] steps repel each other. On the other hand, $\zeta_{3}<0$ for all six elements. This indicates that this $1 / R^{2}$ repulsion between steps is softened at small step-step separations. For sufficiently small $R$, the steps may even attract. However, at these small separations, the expansion in $1 / R$ may not be valid.

Fig. 7a shows the variation of the surface energy per period in $\mathrm{Au}, \Gamma=R \gamma_{01 n}-\left(n a_{0} \gamma_{001}\right) / 2$, with the [100] step spacing $R$ as determined from the simulation data and by fitting Eq. (7) with $k_{\max }=2$ and 3 . This figure shows that the $k_{\max }=2$ functional form provides an excellent fit to the simulation data and the improvement found by including the $(1 / R)^{3}$ term in the step-step interaction energy is small. Similar results were obtained for the other five elements.

The $(11 \mathrm{~m})$ surface energies were fit to Eq. (7) in the same manner as for the $(01 n)$ surfaces and the results are summarized in Table 2 . In this case, the fit only included the $(\overline{1} 1 \mathrm{~m})$ surface energy data for odd $m$ in the range $9 \leq m \leq 25$. The even $m$ data were omitted since they correspond to surfaces with uneven step spacing. Again, we find that the $k_{\max }>2$ functional forms are in better agreement with the $(\overline{1} 1 \mathrm{~m})$ surface energies determined from the simulations than when $k_{\max }=2$. Nonetheless, we find that the $(1 / R)^{2}$ functional form for the step-step interaction also provides an excellent fit to the data (see Fig. 7b) for all six metals examined.

The surface energy fits performed above also provide an estimate of the step energy per unit length for both the [100] and [110] steps on the (001) surfaces in the six elements. These energies are presented in Table 3 along with their corresponding values for the unrelaxed step structure. The step relaxation energies per unit length,
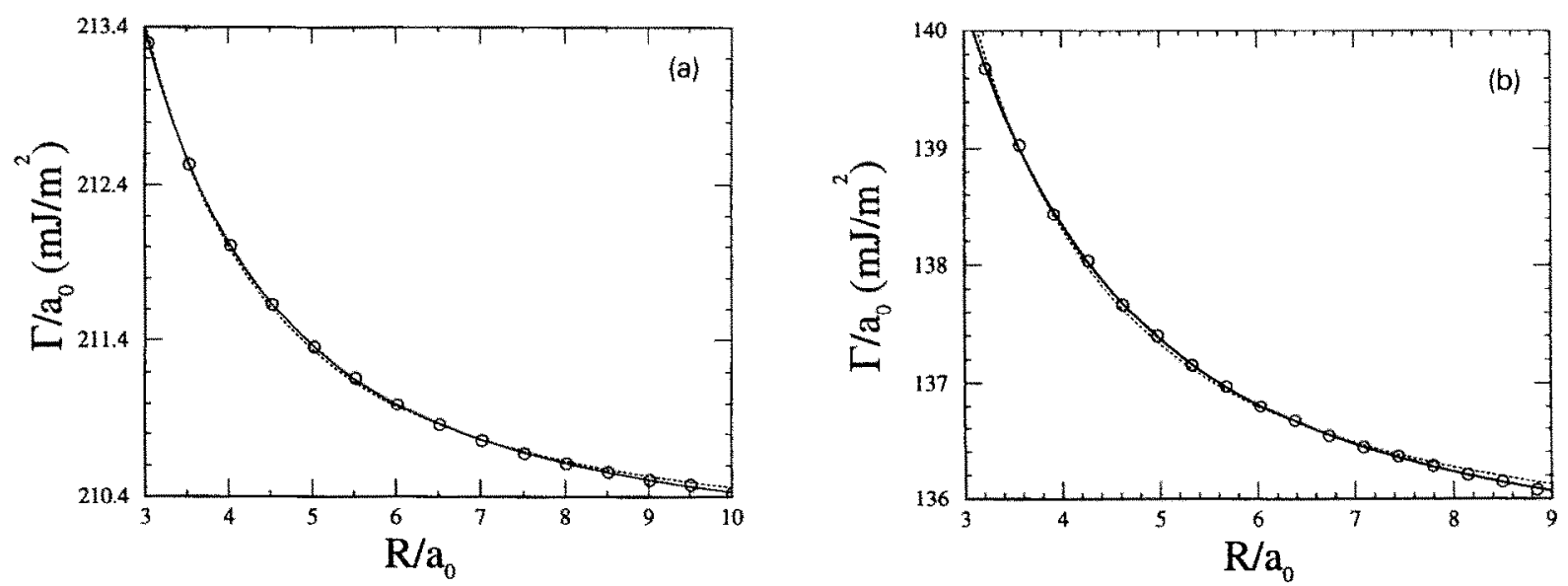

Fig. 7. (a) The variation in the Au[100] step energy, $\Gamma=R \gamma_{01 n}-\left(n a_{0} \gamma_{001}\right) / 2=\gamma_{\text {step }}^{[100]}+\gamma_{\text {int }}(R)$ with step spacing, $R$. The circles represent the atomistic simulation results while the dashed and solid lines are the results of fitting these data to Eq. (7) with $k_{\max }=2$ and 3 , respectively. (b) Shows similar results for the Au(11m) surfaces. Note that in both cases the fit is much better for $k_{\max }=3$ than for $k_{\max }=2$. 
Table 1

The material dependent coefficients $\zeta_{k}$ of the $R^{-k}$ terms in the expansion of the interaction energy between [100] steps (Eq. (7)) extracted from fitting the simulated (01n) surface energies; The $\zeta_{k}$ are reported for fits to the expansion with $k_{\text {max }}=2,3$ and 4 for each of the six foc metals examined; The goodness of fit parameter $\chi^{2}$ would be zero for a perfect fit

\begin{tabular}{|c|c|c|c|c|}
\hline & $\begin{array}{l}10^{13} \zeta_{2} \\
(\mathrm{~J} / \mathrm{m})\end{array}$ & $\begin{array}{l}10^{13} \zeta_{3} \\
(\mathrm{~J} / \mathrm{m})\end{array}$ & $\begin{array}{l}10^{13} \zeta_{4} \\
(\mathrm{~J} / \mathrm{m})\end{array}$ & $x^{2}$ \\
\hline \multirow[t]{3}{*}{$\mathrm{Ag}$} & 19 & & & 0.0013 \\
\hline & 20 & -7 & & 0.0003 \\
\hline & 20 & -3 & -7 & 0.0003 \\
\hline \multirow[t]{3}{*}{ Au } & 73 & & & 0.0271 \\
\hline & 88 & -60 & & 0.0022 \\
\hline & 85 & -34 & -49 & 0.0021 \\
\hline \multirow[t]{3}{*}{$\mathrm{Cu}$} & 41 & & & 0.0014 \\
\hline & 47 & -23 & & 0.0007 \\
\hline & 46 & -14 & -18 & 0.0006 \\
\hline \multirow[t]{3}{*}{$\mathrm{Ni}$} & 26 & & & 0.0027 \\
\hline & 28 & -10 & & 0.0003 \\
\hline & 28 & -5 & -11 & 0.0003 \\
\hline \multirow[t]{3}{*}{ Pd } & 87 & & & 0.0278 \\
\hline & 101 & -55 & & 0.0032 \\
\hline & 97 & -22 & -62 & 0.0030 \\
\hline \multirow[t]{3}{*}{$\mathrm{Pt}$} & 146 & & & 0.0565 \\
\hline & 165 & -74 & & 0.0081 \\
\hline & 161 & -40 & -65 & 0.0078 \\
\hline
\end{tabular}

$\Delta \gamma_{\text {step }}$, defined as the difference in the relaxed and unrelaxed step energies (Table 3), show the same trend as that observed for the flat (001)
Table 2

The material dependent coefficients $\zeta_{k}$ of the $R^{-k}$ terms in the expansion of the interaction energy between [110] steps (Eq. (7)) extracted from fitting the simulated $(\overline{1} 1 \mathrm{~m})$ surface energies

\begin{tabular}{|c|c|c|c|c|}
\hline & $\begin{array}{l}10^{13} \zeta_{2} \\
(\mathrm{~J} / \mathrm{m})\end{array}$ & $\begin{array}{l}10^{13} \zeta_{3} \\
(\mathrm{~J} / \mathrm{m})\end{array}$ & $\begin{array}{l}10^{13} \zeta_{4} \\
(\mathrm{~J} / \mathrm{m})\end{array}$ & $x^{2}$ \\
\hline \multirow[t]{3}{*}{$\mathrm{Ag}$} & 26 & & & 0.0003 \\
\hline & 36 & -34 & & 0.0002 \\
\hline & 34 & -16 & -35 & 0.0002 \\
\hline \multirow[t]{3}{*}{$\mathrm{Au}$} & 108 & & & 0.0077 \\
\hline & 142 & -141 & & 0.0004 \\
\hline & 135 & -78 & -122 & 0.0003 \\
\hline \multirow[t]{3}{*}{$\mathrm{Cu}$} & 56 & & & 0.0031 \\
\hline & 78 & -92 & & 0.0017 \\
\hline & 72 & -48 & -85 & 0.0017 \\
\hline \multirow[t]{3}{*}{$\mathrm{Ni}$} & 36 & & & 0.0007 \\
\hline & 48 & -60 & & 0.0004 \\
\hline & 44 & -29 & -60 & 0.0004 \\
\hline \multirow[t]{3}{*}{$P d$} & 126 & & & 0.0073 \\
\hline & 165 & -166 & & 0.0004 \\
\hline & 156 & -97 & -134 & 0.0004 \\
\hline \multirow[t]{3}{*}{$\mathrm{Pt}$} & 206 & & & 0.0138 \\
\hline & 263 & -255 & & 0.0015 \\
\hline & 247 & -123 & -254 & 0.0016 \\
\hline
\end{tabular}

surface relaxation energies. Specifically, the relaxation is smallest for $\mathrm{Ni}$ and largest for $\mathrm{Pt}$. The ratio of the [100] step energy to that for [110] steps varies from 1.30 for $\mathrm{Cu}$ to 1.56 for $\mathrm{Au}$.

Table 3

The unrelaxed and relaxed step energy and the change in step energy upon relaxation of [100] and [110] steps on the (001) surface in six different fcc metals; The ratio of the [100] and [110] step energies and the change in the energy of the system upon transforming a straight [100] step into a zig-zagged [110] step (Eq. (15)) are also reported

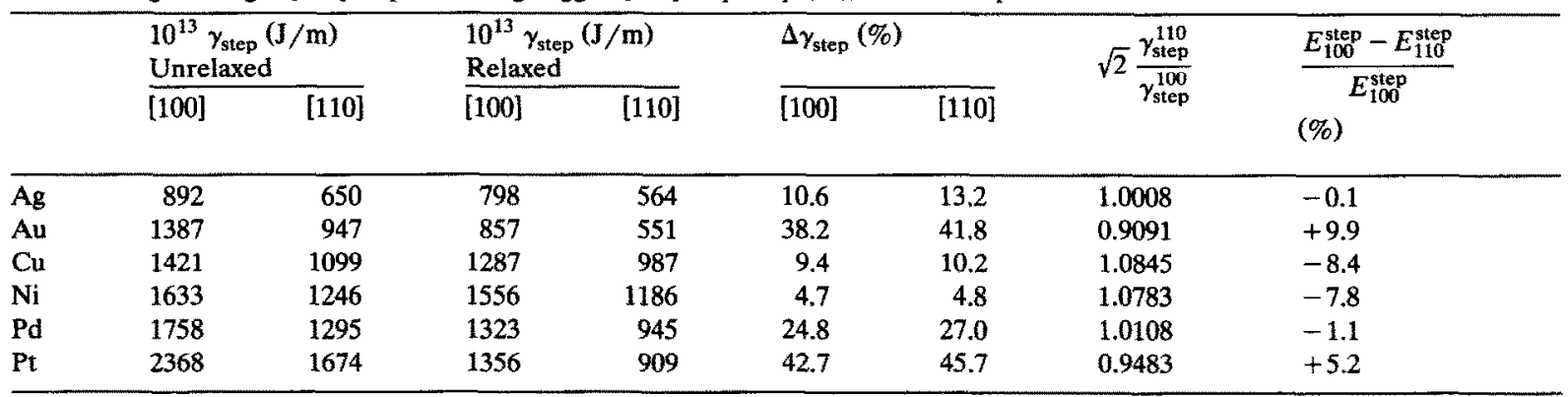




\section{Discussion}

The interaction between [100] steps and between [110] steps on the (001) surfaces of $\mathrm{Ag}, \mathrm{Au}$, $\mathrm{Cu}, \mathrm{Ni}, \mathrm{Pd}$, and $\mathrm{Pt}$ were determined by fitting the surface energies of $(01 n)$ and the $(11 m)$ surfaces to a functional form that separates the terrace, step and step-step interaction energies. The dependence of the step-step interaction energy on step spacing was analyzed by expanding the step-step interaction energy in powers of the interstep spacing $R$. In agreement with the predictions of a continuum elastic theory [1], the step-step interaction energy was found to be proportional to the inverse step spacing $R^{-2}$ for large step spacings. This is also in agreement with the results of a recent atomistic study of the interaction energy between the [100] steps on the (001) surface and on the (011) surface in $\mathrm{Au}$ and $\mathrm{Cu}$ modeled employing the EAM and LennardJones (L) interatomic potential, respectively [3]. However, the fact that the fit between the simulation data and expansions of the step-step interaction energy truncated at higher order (see the $\chi^{2}$ values in Tables 1 and 3 ) was better than that obtained simply with the predicted $R^{-2}$ step-step interaction energy, demonstrates that higher order terms (e.g. $R^{-3}$ ) are important for the range of step separations considered $(6 \leq n \leq 20)$. In some cases, including an $R^{-3}$ term in the stepstep interaction energy decreased $\chi^{2}$ by over an order of magnitude. Inclusion of higher order terms, such as $R^{-4}$ did not further improve the goodness of fit.

The term in the step-step interaction energy proportional to $R^{-3}$ must dominate the $R^{-2}$ term at small step separations and, therefore, the simple continuum elastic theory should be invalid at small step separations. This failure of the simple continuum model has two distinct origins: (1) the neglect of the discrete atomistic nature of the surface and surface steps and (2) the simplified assumption that the elastic field of a step is equivalent to that produced by a surface force dipole. The first problem can only be overcome with an atomistic description of the step. The second can be rectified by performing a multipole expansion of the force distribution that includes

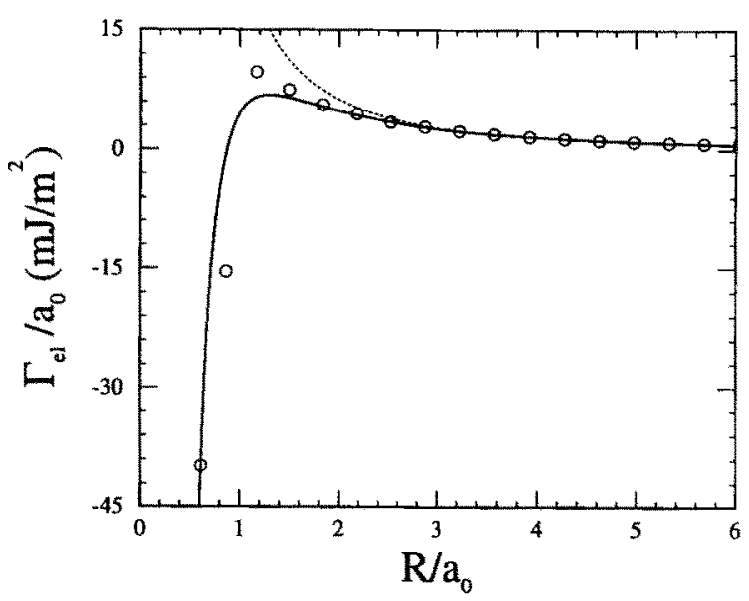

Fig. 8. The elastic component of the step energy $I=R \gamma_{01 n}-$ $\left(n a_{0} \gamma_{001}\right) / 2=\gamma_{\text {step }}^{[100]}+\gamma_{\text {int }}(R)$ for [110] steps on the $(001) \mathrm{Cu}$ surface as a function of step spacing, $R$. The circles represent the energies determined based on our atomistic simulation results while the dashed and solid lines are calculated using the parameters found in fitting the $(11 \mathrm{~m})$ surface energies to Eq. (7) with $k_{\max }=2$ and 3 , respectively.

higher order terms than the simple dipole. A formalism for performing such a multipole expansion has recently been put forward [8]. However, even here, atomistic simulations are required in order to determine the amplitude of the individual terms in the multipole expansion.

In order to further demonstrate the importance of the higher order terms in determining the step-step interaction energy at small step spacing, we plot (Fig. 8) the Cu simulation data and the $k_{\max }=2$ and $k_{\max }=3$ predictions for the step contribution to the surface energy $(\Gamma=$ $\left.R \gamma_{01 n}-\left(n a_{0} \gamma_{001}\right) / 2=\gamma_{\text {step }}^{[100]}+\gamma_{\text {int }}(R)\right)$ for very small step spacings. The $k_{\max }=2$ predictions are in poor agreement with the simulation data at very small step separations, while inclusion of the $R^{-3}$ term captures the essential behavior at small step separations. We observe similar behavior for the other metals considered in the present study. Therefore, it is not reasonable to model step-step interactions using the classical $R^{-2}$ behavior for step spacings down to approximately $a_{0}$, in contradiction to statements in another, recent atomistic study [3]. The good fit between the $k_{\max }=3$ results and the simulation data is impressive because the sharp change in the $\Gamma$ versus $R$ plot 
near $R / a_{0}=1$ was predicted based upon fits obtained for $R \geq 3 a_{0}$. Note that $\gamma_{\text {int }}$, as described in Eq. (3), is the elastic energy due to the interaction among the displacement fields associated with the steps on the surface while that determined based on Eqs. (1) or (2) also include energy due to the formation of bonds between atoms in the steps. This distinction is usually unimportant, but when step spacing is very small (for the EAM potentials used in present study for step spacing less than approximately $1.5 a_{0}$ ) this bonding energy does contribute to $\Gamma$. In Fig. 8, the interaction energy was corrected for this bond formation energy by subtracting the bonding contribution, as determined from the unrelaxed surface energy and Eqs. (1) or (2).

The simple, continuum elasticity analysis of step-step interactions [1] was based on the assumption that the displacement field associated with a single step on an otherwise flat surface can be approximated by that produced by an array of double (dipole) forces acting parallel to the terrace and directed perpendicular to the step: $f_{x} \delta(z) \partial\left[\delta\left(x-x_{0}\right)\right] / \partial x$, where $f_{x}$ is the strength of the force, $\delta(z)$ is a delta function and the step is located at $x=x_{0}$ and runs parallel to the $y$-axis. The real distribution of forces is considerably more complex: it has a finite width, has components into and out of the surface plane [7] and contains in-plane components that are non-dipole in nature. While inclusion of all of these effects is beyond the scope of the present research, we may account for the non-dipole character of the elastic distribution by expanding the true force distribution using a multipole expansion, analogous to the expansion used in the electrostatic theory to describe the electrostatic potential of a charge distribution in terms of its multipole moments [8]. The expansion given in Eq. (7) for the interaction between two steps may be derived from a multipole expansion of the double force distribution associated with a single step. The first term of the expansion in Eq. (7) represents the interaction between the dipole moments of the interacting steps and the higher terms represent the interaction between higher order multipoles. For example, the $R^{-3}$ contribution to the step-step interaction energy is attributable to a dipole- quadrupole interaction and the $R^{-4}$ term to a quadrupole-quadrupole interaction. The fact that the simple continuum elasticity analysis [1] only included the dipole-dipole interaction and was in excellent agreement with the simulation data for all $R>3 a_{0}$, shows that the dipole-dipole interaction energy is clearly the dominant term at these step spacings. However, the significant difference between the simple elastic analysis [1] and our simulation results for the interaction energy observed for $R<1.5 a_{0}$, demonstrates the importance of the dipole-quadrupole $\left(R^{-3}\right)$ interaction in this step-spacing range. Since no improvement in the fit between the simulation data and $\mathrm{Eq}$. (7) was obtained when $k_{\max }$ was increased from 2 to 3 , we conclude that to within the numerical accuracy of our results, multipole interactions of a higher order than dipolequadrupole are unimportant.

The elastic interaction energy between two steps is obtained as an integral over the strain fields due to the individual steps minus that due to widely separated steps. Therefore, in order to further examine the relationship between our simulation results and the elastic theory more closely, we now directly examine the strain field. For a linear elastic medium, the displacement field along a flat surface due to an array of dipole forces acting in the $x$-direction may be written as [9]

$u_{x}(x, z=0)=\frac{2\left(1-\nu^{2}\right)}{\pi E} \frac{f_{x}}{x}$,

where $E$ and $\nu$ are the Young's modulus and the Possion's ratio of the medium, respectively. The strain field $\epsilon_{x x}(x, z=0)$ is simply $\partial u_{x} / \partial x$

$\epsilon_{x x}=(x, z=0)=\frac{2\left(1-\nu^{2}\right)}{\pi E} \frac{f_{x}}{x^{2}}=\frac{A}{x^{2}}$,

where $A$ is a constant.

The total $\epsilon_{x x}^{\text {tot }}$ strain due to a periodic array of steps on the surface with the step spacing $R$ is

$$
\begin{aligned}
\epsilon_{x x}^{\mathrm{tot}}(x) & =\sum_{n=-\infty}^{\infty} \frac{A}{(x-n R)^{2}} \\
& =\frac{\pi^{2} A}{R^{2}}\left\{1+\cot ^{2}\left[\pi\left(1+\frac{x}{R}\right)\right]\right\} .
\end{aligned}
$$




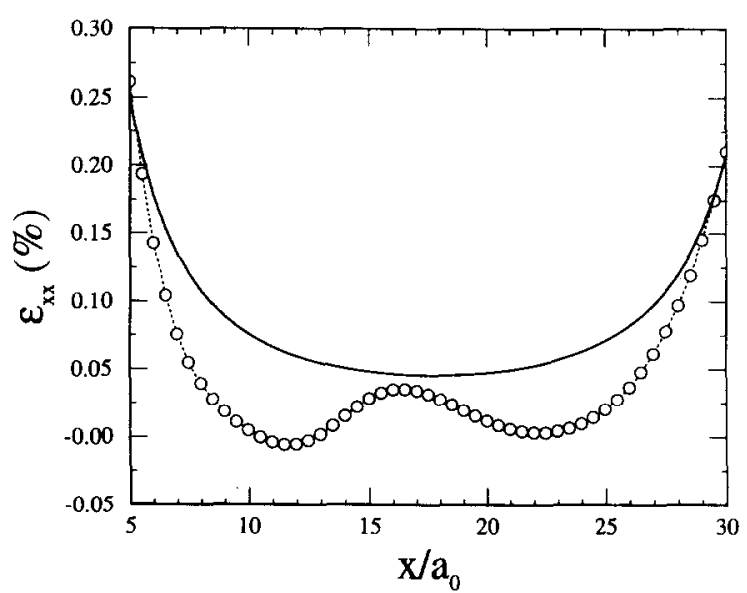

Fig. 9. The $x$ component of the normal strain $\epsilon_{x x}$ on the Au(0171) surface along the $x$ direction (see Fig. 1). The circles are the results of the atomistic simulation data and the solid line represents the results of modeling the step as a line force dipole (see Eq. (14)).

Fig. 9 shows the strain $\epsilon_{x x}^{\text {tot }}$ on the (0171) surface in $\mathrm{Au}$ as calculated numerically from the displacement field determined by our atomistic simulation results and that predicted by the continuum elasticity analysis as given in Eq. (14). The strain $\epsilon_{x x}^{\text {tot }}$ on the surfaces of other metals considered in the present study shows similar behavior. The value of parameter $A$ in Eq. (14) was chosen to yield the same strain value at $x=5 a_{0}$ as that determined in the atomistic simulation results. The (0171) surface was chosen for this comparison since the [100] step spacing on this surface is large $(145 \AA)$, such that the assumption of linear elasticity and the dipole-dipole nature of the interaction should be most applicable, compared with the lower index surfaces in this study. Fig. 9 shows that there are qualitative differences between the results of the two methods for determining $\epsilon_{x x}^{\text {tot }}$. The continuum elastic (dipole-dipole) analysis predicts that $\epsilon_{x x}^{\text {tot }}$ has a mirror symmetry with respect to the $(010)$ plane passing through the midpoints between the steps, that the second derivative of $\epsilon_{x x}^{\text {tot }}$ is everywhere positive and that $\epsilon_{x x}^{\text {tot }}$ itself is positive (tensile) over the entire surface. The symmetry is a result of the periodicity of steps along the $x$ direction and the reflection symmetry in the $x$ direction of the dipole force distribution. However, the atomistic simulation results do not show such a symmetry in the strain field. On the (001) terrace and normal to the step direction, the strain goes through three extrema between the two steps at approximately $x=11.5 a_{0}, 16 a_{0}$, and $22.5 a_{0}$. The (001) terrace experiences compression in the range $10.5 a_{0}<x<13.5 a_{0}$ while it is under tension on the rest of the terrace. The magnitude of the compressive strain on the terrace is, however, relatively small $\left(\epsilon_{x x}^{\text {tot }}<10^{-4}\right)$ on the $\mathrm{Au}(0171)$ surface.

These discrepancies between the dipole elastic theory and the simulation results for the strain field again suggest the importance of the non-dipole terms in defining the elastic fields of the step. However, other possible explanations exist. For example, the effect of elastic anisotropy has not been analyzed and the fcc metals considered here are known to exhibit substantial elastic anisotropy. The anisotropy ratio $2 C_{44} /\left(C_{11}-C_{12}\right)$ (which is unity for no anisotropy) varies from 3.0 in $\mathrm{Ag}$ to 4.5 in Pt. Nonetheless, the main features of the elastic strain field measured in the simulation are reproduced by the dipole elastic theory; namely, the quick decay of the strain field away from the steps and the presence of only very small strains between these widely separated steps. It is important to note that small errors (of order $e$ ) in the strain produce even smaller errors in the strain energy density (of order $e^{2}$ ).

The step energy per unit length for the [100] step on the $A u(001)$ surface is $857 \times 10^{-13} \mathrm{~J} / \mathrm{m}$, in good agreement with $824 \times 10^{-13} \mathrm{~J} / \mathrm{m}$ determined in a recent atomistic simulation study [3] employing the same EAM potential. The small difference $(4 \%)$ in the two energy determinations is presumably attributable to a slight modification made in the EAM potential in that study. However, for the [100] step on the $\mathrm{Cu}(001)$ surface employing the copper EAM potential, we find the step energy to be $1287 \times 10^{-13} \mathrm{~J} / \mathrm{m}$ while it was found to be only $792 \times 10^{13} \mathrm{~J} / \mathrm{m}$ when a Lennard-Jones potential fitted to the copper lattice constant and melting point is used [3]. It was suggested [3] that the remarkable similarity in the step energies $824 \times 10^{-13}$ and $792 \times 10^{-13} \mathrm{~J} / \mathrm{m}$ for $\mathrm{Au}(\mathrm{EAM})$ and $\mathrm{Cu}(\mathrm{LJ})$, respectively, is due to 
the fact that the step energy is dominated by the underlying core contributions (i.e., by the number of broken bonds per unit length of step and, hence, by the short-range repulsion between neighboring atoms) and both the Au(EAM) and $\mathrm{Cu}(\mathrm{LJ})$ have similar repulsive potentials. While both the dominance of core contributions to the step energy and the similarly in the repulsive part of the EAM and $\mathrm{LJ}$ potentials are correct, we find a significant difference (70\%) in the [100] step energies when the same type of interatomic potential (EAM) is used for both $\mathrm{Au}$ and $\mathrm{Cu}$. In addition, we also note (see Table 3 ) that the [100] step energy for the six elements using the EAM potentials changes from $798 \times 10^{-13} \mathrm{~J} / \mathrm{m}$ for $\mathrm{Ag}$ to $1556 \times 10^{-13} \mathrm{~J} / \mathrm{m}$ for $\mathrm{Ni}$. The fact that the step energies are different for different materials as observed in this study, like any other defect energy, is to be expected and is due partly to the difference in the bond strengths of different materials.

Inspection of Table 3 shows that the [110] step energy is approximately $40 \%$ smaller than that of a [100] step on a (001) surface. This difference is largely attributable to the fact that [110] steps have fewer broken bonds per unit length than do [100] steps. If the difference in the step energies is sufficiently large, then it may be energetically favorable for the [100] steps on the (001) surface to transform into [110] steps. However, in order to evaluate the stability of [100] versus [110] steps on a (001) surface, we must consider the difference in the energies of a straight [100] step and a zig-zagged [110] step, as shown in Fig. 10. Note that due to the crystallographic orientations of these steps, the angle between the [100] step and different segments of the zig-zagged [110] step are either $45^{\circ}$ or $135^{\circ}$. If we assume that the energy due to the interactions between different segments of the zig-zagged [110] step is small compared to the step energy, then the difference in the energies of these two steps configurations (per unit length $L$ of the [100] step) is simply

$E_{100}^{\text {step }}-E_{110}^{\text {step }}=L \gamma_{\text {step }}^{100}\left(1-\sqrt{2} \frac{\gamma_{\text {stcp }}^{110}}{\gamma_{\text {step }}^{100}}\right)$,

where the step energy ratios for the six metals are

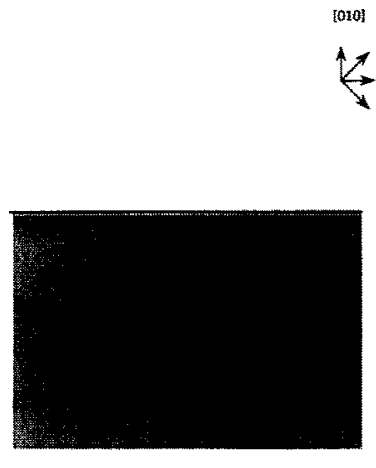

(a)
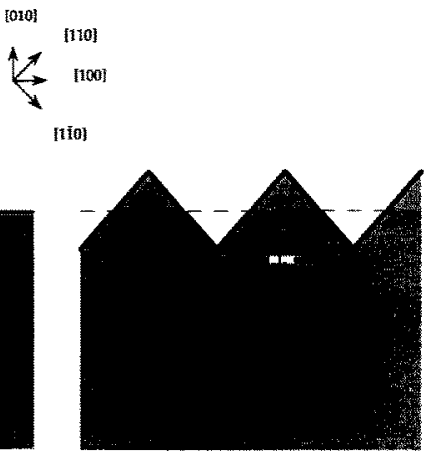

(b)
Fig. 10. A schematic illustration of a (001) surface (seen from above) with (a) a straight [100] step and (b) a zig-zagged [110] step. The shaded region represents part of the upper terrace.

given in Table 3 . The (001) surface energy doesnot enter into this equation since the surface area remains unchanged upon this transformation.

The difference in energy between a straight [001] and a zig-zagged [110] step, Eq. (15), is negative for $\mathrm{Ag}, \mathrm{Cu}, \mathrm{Ni}$, and $\mathrm{Pd}$ while it is positive for both $\mathrm{Au}$ and $\mathrm{Pt}$, as shown in Table 3 . Thus a straight [100] step on the (001) surface in $\mathrm{Au}$ or Pt would lower its energy by transforming to a zig-zagged [110] step while it remains stable on the (001) surfaces of $\mathrm{Ag}, \mathrm{Cu}, \mathrm{Ni}$, and $\mathrm{Pd}$. Although it is difficult, however, to determine the effect of interactions between different segments of the zig-zagged [110] step on this stability analysis, one may crudely approximate this energy by assuming that the average step spacing between the nearest neighbor segments is $L / 2$. Fig. 10 shows that the interaction energy between [110] steps on the Au(001) surface is less than $4 \times 10^{-13}$ $\mathrm{J} / \mathrm{m}$ when $R>5 a_{0}\left(L>10 a_{0}\right)$. This interaction energy is much smaller than $857 \times 10^{-13}$ and $551 \times 10^{-13} \mathrm{~J} / \mathrm{m}$ for the [100] and [110] step energies on the $A u(001)$ surface, respectively, and, therefore, the interaction between different segments of the zig-zagged [110] steps does not alter the above conclusion. It should be noted, however, that the present [100] step stability analysis has not considered the entropic contribution to the step energy (free energy) and, therefore, the present prediction applies to sufficiently low temperatures where the entropic contribution is 
small. A similar analysis for the transformation of a straight [110] step into a zig-zagged [100] step on a (001) surface, shows that the straight [110] step is stable against transforming into [110] steps for all six fcc metals examined in this study.

\section{Summary and conclusions}

We have employed an energy minimization method $(T=0)$ and the EAM potentials to determine the $(01 n)$ and $(11 \mathrm{~m})$ surface energies for $n \leq 71$ and $m \leq 41$, respectively, for the elements: $\mathrm{Ag}, \mathrm{Au}, \mathrm{Cu}, \mathrm{Ni}, \mathrm{Pd}$, and Pt. An elastic model is used to extract step-step interaction energies between [100] steps and between [110] steps on the (001) surface of these fcc metals.

- For step spacings larger than approximately three fcc lattice parameters $\left(R>3 a_{0}\right)$, the interaction energies between [100] steps and between [110] steps on the (001) surface in the six metals can be reasonably described as being proportional to $R^{-2}$. This is in agreement with a simple linear elastic theory of step interactions $[1,7,8]$ and a recent atomistic simulation study [3]. However, a detailed comparison of the step strain fields implicit in the elastic theory with that predicted by the simulations, shows that modeling steps as in-surface-plane dipole line forces in an isotropic elastic medium leads to the prediction of elastic fields that are in qualitative and quantitative disagreement with simulation results.

- For interstep spacings smaller than three fcc lattice parameters $\left(R<3 a_{0}\right)$, we find large differences between the step-step interaction energies predicted by the simple linear elastic theory $[1,7,8]$ and the atomistic simulations for both [100] and [110] steps on (001) surfaces. In particular, we find significant deviations from the $R^{-2}$ functional form predicted by the elastic dipole force model for surface steps $[1,8]$. Expanding the step-step interaction energy in powers of $R^{-k}$, shows that in addition to the dipole-dipole contribution which is dominant at large step separation, dipole and quadrupole contributions are important at small step spacing. We find that while dipole-dipole and dipole-quadrupole are important for determining the step-step interac- tion energy, higher order moments are unimportant.

- The interaction between [100] steps are weaker than that between [110] steps on the (001) surface in all six metals. Among the six metals studied here, the interaction between two similar steps on the (001) surface is weakest in $\mathrm{Ag}$ and strongest in $\mathrm{Pt}$.

- A simple comparison of the surface energies of [100] and [110] steps on (001) surfaces, shows that straight [100] steps are unstable to faceting into zig-zagged [110] steps in $\mathrm{Au}$ and $\mathrm{Pt}$, but not in $\mathrm{Ag}, \mathrm{Au}, \mathrm{Cu}, \mathrm{Pd}$ or Ni. Straight [110] steps are stable against faceting into zig-zagged $[100]$ steps on (001) surfaces of all six fcc metals examined.

\section{Acknowledgments}

The authors thank Professor J.M. Rickman for useful discussions on the subject of this paper. D.J.S. gratefully acknowledges the hospitality of The Weizmann Institute of Science and the support of the Michael Visiting Professorship. This research was supported by the Division of Materials Science of the Office of Basic Energy Sciences of the U.S. Department of Energy, Grant No. FG02-88ER-45367.

\section{References}

[1] V.I. Marchenko and A.Ya. Parshin, Sov. Phys. JETP 52 (1981) 129

[2] P. Wynblatt, in: Interatomic Potentials and Simulation of lattice Defects, Eds. P. Gehlen, I.R. Beeler, IT. and R.I. Jaffee (Plenum, New York, 1972).

[3] D. Wolf and J.A. Jaszczak, Surf. Sci. 277 (1992) 301.

[4] T.W. Poon, S. Yip, P.S. Ho and F.F. Abraham, Phys. Rev. Lett. 65 (1990) 2161.

[5] S.M. Foiles, M.I. Baskes and M.S. Daw, Phys. Rev. B 33 (1986) 7983 .

[6] R. Najafabadi, D.J. Srolovitz and R. LeSar, J. Mater. Res, 5 (1990) 2663.

[7] D.J. Srolovitz and J.P. Hirth, Surf. Sci. 255 (1991) 111.

[8] J.M. Rickman and D.J. Srolovitz, Surf. Sci. 284 (1993) 211.

[9] P. Nozieres, in: Lecture given at the Beg-Rohu Summer School (1989) 45.

[10] J.D. Jackson, in: Classical Electrodynamics (Wiley, New York, 1975). p. 136. 